\title{
Man-made chemicals and reproductive health
}

There is plenty of circumstantial evidence to link synthetic chemicals that mimic oestrogens to falling sperm counts, abnormalities of the male reproductive system and increases in the number of cases of breast cancer, according to a UK government report published in late July.

The report, Environmental Oestrogens: Consequences to Human Health and Wildlife, authored by Lewis Smith et al., director of the Medical Research Council's Institute for Environment and Health in Leicester and colleagues, provides a review of all the research on links between chemicals that mimic the effects of oestrogen, or that block the effects of androgen, and reproductive health. It considers the evidence for a decline in sperm counts and the motility of sperm, whether man-made chemicals do in fact mimic oestrogen, and the effects of high doses of these chemicals on the reproductive systems of animals.

The report concludes that there is no proof of a causal link between chemical pollution and reproductive health problems in humans. Although without such proof there is no justification for an immediate ban on any specific chemicals, the circumstantial evidence means further research is warranted.

The main classes of chemicals implicated are phthalates, which are used in food packaging as well as in dyes, insecticides, and pharmaceuticals; alkylphenols used in the production of industrial detergents; and organochlorine pesticides including DDT, which is already banned in most developed countries.

In spite of conflicting data, Smith believes on balance that there has been a fall in sperm count and sperm quality over the past 50 years, and a rise in cases of cryptorchidism (undescended testicles), testicular cancer and hypospadias (malformation of the penis), as well as an increase in breast cancer in males and females.

Various studies indicate that sperm counts worldwide have fallen by 2 per cent a year, and that the number of mobile sperm has fallen by 1 per cent a year over the past 20 to 30 years. At the same time, the incidence of testicular cancer has increased by more than 30 per cent, while breast cancer in females has risen by 25 to 30 per cent since the 1940s. However, Smith says there is "no convincing evidence" of a concomitant decrease in fertility.

Although there is no concrete evidence

\author{
IMAGE \\ UNAVAILABLE \\ FOR COPYRIGHT \\ REASONS
}

Electronmicrograph of an abnormal extrusion developing near the spermatazoon head.

that chemical pollution causes these problems in humans, there is laboratory and field research that is suggestive of this. Observations of so-called gender bending effects include alligators with stunted genitalia, male fish becoming hermaphroditic and female mollusks with penises. Smith points out that showing an effect at high doses in animals does not prove that low-dose exposure will harm humans.

The proposed research into possible links between trends in reproductive health and exposure to chemicals with known oestrogenic or related hormonal effects will do the following:

- Examine the extent of human exposure to oestrogen mimics,

- Develop a test for chemicals that can interfere with the action of natural oestrogen in the body,

- Develop a standardized national approach to measuring sperm counts and assessing sperm quality,

- Study the development of the male fetus in the first trimester to ascertain if oestrogen mimics can influence the development of the testes,

- Undertake epidemiological studies of testicular and breast cancer.

Nuala Moran London

\section{Australian gene technology survey}

Most Australians approve of the use of genetic engineering, with a person's social background having little effect on its acceptance, according to a recent study commissioned by the government and carried out by researchers at the Australian National University in Canberra.

The study, Public Perceptions of Genetic Engineering: Australia, 1994, examined the attitudes of more than 1,350 individuals. All the states and territories in Australia were represented in the sample. In addition to the usual demographic data, such as age, religion, political persuasion, respondents were asked about their attitudes towards scientific research in general, their approval of the use of genetic engineering in agricultural products and medicine in particular, their level of concern about the potential risks posed by genetic engineering, and whether they favoured the labelling of genetically modified products.

The study found that most Australians claim to have at least a basic understanding of science and accept the so-called scientific world view. There was broad support for the use of genetic engineering in medical applications. Respondents also favoured widespread use of the technology in agriculture, which was the main focus of the study. However, they also wanted genetically altered products to be clearly labelled. According to the study's authors, a small minority rejected both the scientific world view and genetic engineering.

With respect to the risks associated with genetically engineered products, many respondents worried about the possible emergence of new diseases. However, the study's authors say the level of concern is on a par with that surrounding the use of chemical pesticides. Overall, the study found that most Australians believe the long-term benefits of genetic engineering far outweigh its potential risks.

The study was commissioned by the Department of Industry, Science and Technology in an effort by the Australian government to verify anecdotal claims of national attitudes towards gene technology and to have a systematic reference for comparison with other countries.

Preliminary results from the study received mixed reviews when they were presented at the 4th Pacific Rim Biotechnology Conference held in Melbourne last February. Questions were raised about possible bias in the questions towards an optimistic outcome. The full report will be submitted for peer review. Government officials, who received it in May, hope to use the findings to draw up legislation for the Australian gene technology industry, which is now govemed by voluntary guidelines.

ELZABETH BAN Sydney 